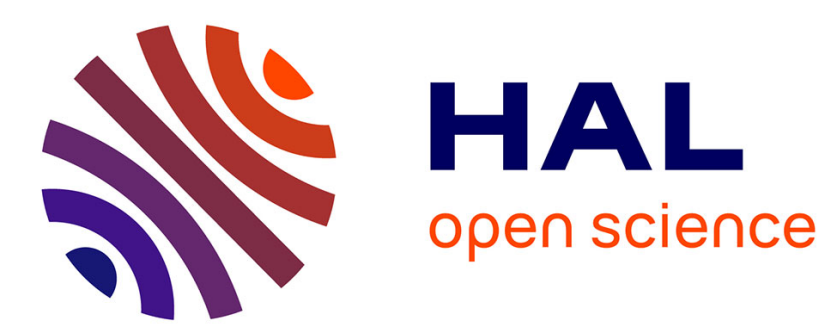

\title{
Numerical simulations of a fluid-particle coupling
}

Nina Aguillon

\section{To cite this version:}

Nina Aguillon. Numerical simulations of a fluid-particle coupling. Finite Volumes for Complex Applications VII, Jun 2014, Berlin, Germany. 10.1007/978-3-319-05591-6 . hal-00986277

\section{HAL Id: hal-00986277 https://hal.science/hal-00986277}

Submitted on 2 May 2014

HAL is a multi-disciplinary open access archive for the deposit and dissemination of scientific research documents, whether they are published or not. The documents may come from teaching and research institutions in France or abroad, or from public or private research centers.
L'archive ouverte pluridisciplinaire HAL, est destinée au dépôt et à la diffusion de documents scientifiques de niveau recherche, publiés ou non, émanant des établissements d'enseignement et de recherche français ou étrangers, des laboratoires publics ou privés. 


\title{
Numerical simulations of a fluid-particle coupling
}

Nina Aguillon

\begin{abstract}
We present numerical simulations of a model of coupling between a inviscid compressible fluid and a pointwise particle. The particle is seen as a moving interface, through which interface conditions are prescribed. Key points are to impose those conditions at the numerical level, and to deal with the coupling between an ordinary and a partial differential equations.
\end{abstract}

\section{The model}

We consider the following coupling, introduced in [2], between a pointwise particle of position $h$, and a fluid governed by the isothermal Euler equations, having density $\rho(t, x)$ and velocity $u(t, x)$ at time $t$ and point $x$ :

$$
\left\{\begin{array}{l}
\partial_{t} \rho+\partial_{x}(\rho u)=0 \\
\partial_{t}(\rho u)+\partial_{x}\left(\rho u^{2}+c^{2} \rho\right)=-D\left(\rho, \rho\left(u-h^{\prime}(t)\right)\right) \delta_{h(t)}(x) \\
m h^{\prime \prime}(t)=D\left(\rho(t, h(t)), \rho\left(u(t, h(t))-h^{\prime}(t)\right)\right)
\end{array}\right.
$$

Here, $c$ is the speed of sound. The fluid and the particle interact with each other through the drag force $D$, which applies only at the point where the particle is located. If $D$ has the same sign as $u-h^{\prime}$, it formally tends to bring the velocities of the fluid and the particle closer to each other. Indeed the third line of (1), which is nothing else than Newton's law applied to the particle, yields that the particle accelerates if its velocity is smaller than the fluid's velocity. This system is a generalization of the coupling between a particle and an inviscid fluid introduced and studied in [15], [5] and [4] (see references therein). In [6], one can find another model of coupling between a pointwise particle and a compressible inviscid fluid.

Nina Aguillon

Université Paris Sud, F-91405 Orsay, France, e-mail: nina.aguillon @ math.u-psud.fr 
The model is different, and local in time existence of solution for small subsonic data is proved, with tools develop in [8]. Let us start with two remarks about System (1). We denote by $H$ the Heaviside function. With the new unknown $w:=H(x-h(t))$, which verifies $\partial_{t} w-h^{\prime}(t) \partial_{x} w=0$, we can write (1) as a non-conservative system of conservation laws. It is not strictly hyperbolic: its Jacobian matrix has eigenvalues $u-c, u+c$ and $h^{\prime}$, and is not diagonalizable when $h^{\prime}=u \pm c$. Moreover, as shocks appear in finite time in the solutions of the Euler equations, the right hand-sides of (1) are not well defined. However, it is possible to reformulate the System (1) as an interface problem. In the sequel we denote by $\left(\rho_{-}, u_{-}\right)$and $\left(\rho_{+}, u_{+}\right)$the traces of the fluid on the left and on the right of the particle: e.g. $\rho_{-}(t)=\lim _{x \rightarrow h(t)-} \rho(t, x)$. Interface conditions are imposed by saying that the traces must belong to a certain set. In the spirit of [3], we call that set the germ and we denote it by $\mathscr{G}_{D}\left(h^{\prime}\right)$.

Definition 1. We denote by $F_{\alpha}$ an antiderivative of the function $\rho \mapsto \frac{\alpha^{2} / \rho+c^{2} \rho}{|D(\rho, \alpha)|}$. The germ $\mathscr{G}_{D}\left(h^{\prime}\right)$ is the set of $\left(\left(\rho_{-}, u_{-}\right),\left(\rho_{+}, u_{+}\right)\right)$in $\left(\mathbb{R}_{+} \times \mathbb{R}\right)^{2}$ such that

1. $\rho_{-}\left(u_{-}-h^{\prime}\right)=\rho_{+}\left(u_{+}-h^{\prime}\right)$. We denote by $\alpha$ this quantity;

2. Either $F_{\alpha}\left(\rho_{-}\right)-F_{\alpha}\left(\rho_{+}\right)=\operatorname{sign}(\alpha)$, or there exists $\theta \in[0,1]$ and $\rho_{0} \leq \frac{|\alpha|}{c}$ such that
a. $\rho_{-} \leq \frac{\alpha}{c} \leq \rho_{+}$and $F_{\alpha}\left(\rho_{-}\right)-F_{\alpha}\left(\rho_{0}\right)=\theta$ and $F_{\alpha}\left(\frac{c^{2}}{\alpha^{2} \rho_{0}}\right)-F_{\alpha}\left(\rho_{+}\right)=(1-\theta)$;
b. $\rho_{+} \leq \frac{-\alpha}{c} \leq \rho_{-}$and $F_{\alpha}\left(\rho_{+}\right)-F_{\alpha}\left(\rho_{0}\right)=\theta$ and $F_{\alpha}\left(\frac{c^{2}}{\alpha^{2} \rho_{0}}\right)-F_{\alpha}\left(\rho_{-}\right)=(1-\theta)$;

3. If $u_{-}>h^{\prime}$ and $u_{-}-h^{\prime} \leq c$, then $u_{+}-h^{\prime} \leq c$;

4. If $u_{+}<h^{\prime}$ and $u_{+}-h^{\prime} \geq-c$, then $u_{-}-h^{\prime} \geq-c$.

This relation are obtained thanks to a thickening of the particle, where the Heaviside function $H$ is replaced by one of its regularization $H_{\varepsilon}$. It appears that the densities and velocities at the entry and at the exit of the particle are always linked by the relations of Definition 1, whatever the size $\varepsilon$ of the particle is, and which regularization is chosen (see [2] for more details). The "Riemann invariants" of the wave associated to eigenvalue $h^{\prime}$ of System (1) are $\alpha=\rho\left(u-h^{\prime}\right)$ and $F_{\alpha}-H_{\varepsilon}$.

Definition 2. A triplet $(\rho, u, h) \in L^{\infty}\left(\mathbb{R}_{+} \times \mathbb{R}\right) \times L^{\infty}\left(\mathbb{R}_{+} \times \mathbb{R}\right) \times W_{l o c}^{2, \infty}\left(\mathbb{R}_{+}\right)$is called an entropy solution of the problem (1) if:

1. The pair of functions $(\rho, u)$ is a weak entropy solution of the isothermal Euler equations on the sets $\left\{(t, x) \in \mathbb{R}_{+}^{*} \times \mathbb{R}: x>h(t)\right\}$ and $\left\{(t, x) \in \mathbb{R}_{+}^{*} \times \mathbb{R}: x<h(t)\right\}$;

2. For almost every $t>0$, the traces around the particle exist and belong to the germ at speed $h^{\prime}(t):\left(\left(\rho_{-}(t), u_{-}(t)\right),\left(\rho_{+}(t), u_{+}(t)\right) \in \mathscr{G}_{D}\left(h^{\prime}(t)\right)\right.$;

3. For almost every $t>0$, the particle is driven by the ODE:

$$
m h^{\prime \prime}(t)=c^{2}\left(\rho_{-}(t)-\rho_{+}(t)\right)\left(1-\frac{\left(u_{-}(t)-h^{\prime}(t)\right)\left(u_{+}(t)-h^{\prime}(t)\right)}{c^{2}}\right) .
$$

The following Proposition, which is proven by simple computations, justifies the first point of Definition 1 and the reformulation (2) of the ODE. 
Proposition 1. A solution $(\rho, u)$ of the Euler equation on the sets $\{x<h\}$ and $\{x>$ $h\}$, with total bounded variations, conserves the total mass $\int_{\mathbb{R}} \rho d x$ if and only if for almost every time,

$$
\rho_{-}\left(u_{-}-h^{\prime}\right)=\rho_{+}\left(u_{+}-h^{\prime}\right) .
$$

In that case, it conserves the total impulsion $\int_{\mathbb{R}} \rho u d x+m h^{\prime}$ if and only if for almost every time, the particle is driven by Equation (2).

Proof. The proof consists in cutting integrals on $\mathbb{R}$ as integrals on $\{x<h\}$ and $\{x>h\}$. For the total impulsion, we obtain that $h$ must verify

$$
m h^{\prime \prime}(t)=h^{\prime}\left(\rho_{+} u_{+}-\rho_{-} u_{-}\right)+\left(\rho_{-} u_{-}^{2}+c^{2} \rho_{-}\right)-\left(\rho_{+} u_{+}^{2}+c^{2} \rho_{+}\right) .
$$

When the mass is conserved, we express $u_{ \pm}$in terms of $\rho_{ \pm}$and $\alpha:=\rho_{ \pm}\left(u_{ \pm}-h^{\prime}\right)$ to obtain (2).

The main result of [2] exhibits some conditions under which the Riemann problem for a motionless particle is well-posed.

Theorem 1. Consider a particle having a constant velocity equal to some real $v$. If the drag force $D$ has the same sign as $\alpha:=\rho(u-v)$, is an increasing function of $\alpha$ and if $|D|$ is a decreasing function of $\rho$, then for all $\left(\left(\rho_{L}, u_{L}\right),\left(\rho_{R}, u_{R}\right)\right)$ in $\left(\mathbb{R}_{+} \times \mathbb{R}\right)^{2}$, there exists a unique self similar solution to the Riemann problem

$$
\left\{\begin{array}{l}
\partial_{t} \rho+\partial_{x}(\rho u)=0, \\
\partial_{t}(\rho u)+\partial_{x}\left(\rho u^{2}+c^{2} \rho\right)=-D(\rho, \alpha) \delta_{v t}(x), \\
(\rho(0, x), u(0, x))=\left(\rho_{L}, u_{L}\right) \mathbf{1}_{x<0}+\left(\rho_{R}, u_{R}\right) \mathbf{1}_{x>0}
\end{array}\right.
$$

The main difficulty is the non-hyperbolicity of the system. The Riemann problem has a more complicated structure than in the strictly hyperbolic case, and in particular uniqueness can be lost (see for example [14] and [11]). This is the case for the drag force $D(\rho, \alpha)=\rho$ illustrated below, which violates the hypothesis of Theorem 1. Remark that this source term is similar to the source term in the shallow water equations with discontinuous topography. The Riemann problem (3) with $\rho_{L}=0.7$,
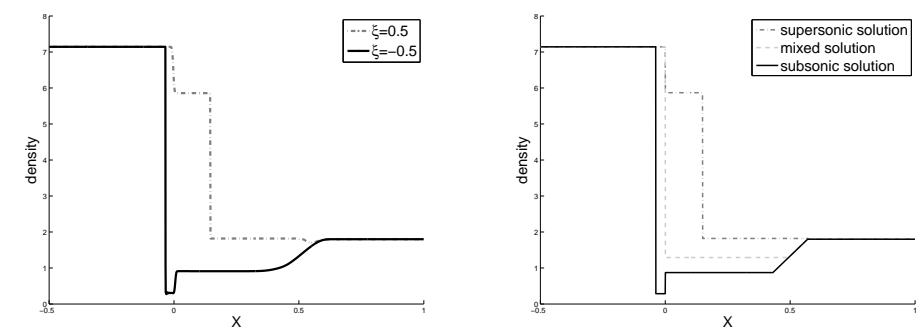

Fig. 1 Left : solutions at time $T=0.15$ given by the Godunov scheme for different regularizations of the Dirac measure. Right: the three solutions of the Riemann problem. 
$\rho_{R}=5, q_{L}=5, q_{R}=9, c=2$ and $\lambda=1.5$ admits three solutions, depicted on the right of Figure 1. As in [7], this coexistence of solutions persists at the numerical level. We can see on the left of Figure 1 two solutions selected by the Godunov scheme when replacing the Dirac measure by

$$
x \mapsto \exp \left((x / \eta-\xi)^{2}\right) /(\eta \sqrt{2}),
$$

with $\eta=0.005$ and $\xi=-0.5$ or $\xi=0.5$. We used a splitting between the fluid part and the regularized source term. The subsonic and supersonic solutions are obtained for large range of parameter $\xi$, with a very quick transition between the two passing through the mixed solution.

\section{Finite volume schemes for the coupled system}

In the sequel, we adopt classical notation for finite volume schemes. We denote by $q=\rho u$ the momentum of the fluid. In particular, $U_{j}^{n}=\left(\rho_{j}^{n}, q_{j}^{n}\right)$ is an approximation of the solution at the $n$-th iteration in time and in the $j$-th cell, and $g$ is the numerical flux. Consider the case where the particle has a fixed constant velocity $v$, and denote by $j_{0}^{n}$ the cell where the particle lies at the $n$-th iteration in time. The three points scheme

$$
\begin{cases}U_{j}^{n+1 / 2} & =U_{j}^{n}-\frac{\Delta t}{\Delta x}\left(g\left(U_{j}^{n}, U_{j+1}^{n}\right)-g\left(U_{j-1}^{n}, U_{j}^{n}\right)\right) \\
U_{j_{0}^{n}}^{n+1} & =U_{j_{0}^{n}}^{n+1 / 2}-\frac{\Delta t}{\Delta x}\left(\begin{array}{c}
0 \\
D\left(\rho_{j_{0}^{n}}^{n+1 / 2}, \rho_{j_{0}}^{n+1 / 2}\left(u_{j_{0}^{n}}^{n+1 / 2}-v\right)\right)
\end{array}\right),\end{cases}
$$

corresponds to a splitting scheme between the evolution of the fluid (first line) and the influence of the particle (second line). This scheme does not converge toward the correct solution, even in the simplest case where $D\left(\rho, \rho\left(u-h^{\prime}\right)\right)=\lambda \rho(u-$ $h^{\prime}$ ) (which fulfills the hypothesis of Theorem 1) and the initial data belongs to the germ. It can be seen on Figure 2. This failure to capture a small scale phenomenon

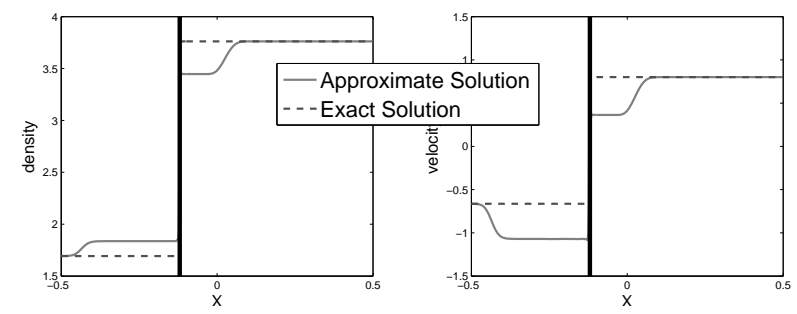

Fig. 2 Solution at time $T=0.04$ given by the fluid-particle splitting. 
recalls the difficulties encountered when approximating non-classical shocks (see for example [13]) or non-conservative systems (see for example [9] and [16]). It illustrates that the reformulation as an interface problem of system (1) is necessary.

\subsection{Schemes for a motionless particle}

When the particle is motionless, we can easily implement schemes based on the exact resolution of the Riemann problem, which is constructed in the proof of Theorem 1. Since the particle is not moving, the particle is a fixed interface that we place between cells numbered 0 and 1. We use a ghost-fluid approach (see [10] and [1]) to write the scheme

$$
\begin{cases}U_{j}^{n+1}=U_{j}^{n}-\frac{\Delta t}{\Delta x}\left(g\left(U_{j}^{n}, U_{j+1}^{n}\right)-g\left(U_{j-1}^{n}, U_{j}^{n}\right)\right) & \text { for } j \notin\{0,1\}, \\ U_{0}^{n+1}=U_{0}^{n}-\frac{\Delta t}{\Delta x}\left(g\left(U_{0}^{n}, U_{\text {part },-}^{n}\right)-g\left(U_{-1}^{n}, U_{0}^{n}\right)\right) & \text { for } j=0, \\ U_{1}^{n+1}=U_{1}^{n}-\frac{\Delta t}{\Delta x}\left(g\left(U_{1}^{n}, U_{2}^{n}\right)-g\left(U_{\text {part },+}^{n}, U_{1}^{n}\right)\right) & \text { for } j=1 .\end{cases}
$$

Here, $U_{\text {part },-}^{n}=\left(\rho_{\text {part },-}^{n}, q_{\text {part },-}^{n}\right)$ and $U_{\text {part },+}^{n}=\left(\rho_{\text {part },+}^{n}, q_{\text {part },+}^{n}\right)$ are the values of the density and the momentum of the fluid on lines $x=0^{-}$and $x=0^{+}$of the unique self similar solution to (3), with

$$
\rho_{L}=\rho_{0}^{n}, u_{L}=\frac{q_{0}^{n}}{\rho_{0}^{n}}, \rho_{R}=\rho_{1}^{n} \text { and } u_{R}=\frac{q_{1}^{n}}{\rho_{1}^{n}} .
$$

Remark that when $g$ is the Godunov flux, $U_{0}^{n+1}$ and $U_{1}^{n+1}$ are the averages of the exact solution with particle given by Theorem 1 . In other words, it is the original Godunov scheme for the fluid/particle coupling. If we start with a Riemann problem belonging to $\mathscr{G}_{D}(0)$, i.e. verifying the relations of Defintion 1 , we obtain for all $n$,

$$
U_{\text {part },-}^{n}=U_{L} \quad \text { and } U_{\text {part },+}^{n}=U_{R} .
$$

Adopting the vocabulary of [12], it follows that the scheme (4) is well balanced with respect to the whole germ $\mathscr{G}_{D}(0)$. We used this scheme to simulate a clogged organ pipe. The pipe is initially filled with a fluid at rest having density $5 \mathrm{~kg} / \mathrm{m}$, and we take $c=1 \mathrm{~m} / \mathrm{s}$. At time $t>0$, a constant flow of $3 \mathrm{~kg} / \mathrm{s}$ is imposed on the left entry of the pipe, while the gas exits freely on the right. The pipe is blocked in its middle by a porous particle that we model using the drag force $D(\rho, \rho u)=\rho u$. At time $0.041 s$, the shock emitted by the left boundary condition hits the particle. The Riemann problem with the particle develops one shock on each side of the particle. Roughly speaking, most of the air is stuck in front of the particle, causing an elevation of its density and a decrease of its velocity. A small part of the fluid manages to pass through the particle, and has a large velocity on the exit of the particle by conservation of momentum through the particle. The shock on the left of the particle interacts with the left boundary at time $0.114 \mathrm{~s}$, creating another shock that meets the particle at time $0.153 \mathrm{~s}$. Asymptotically, the fluid has constant momentum all over 

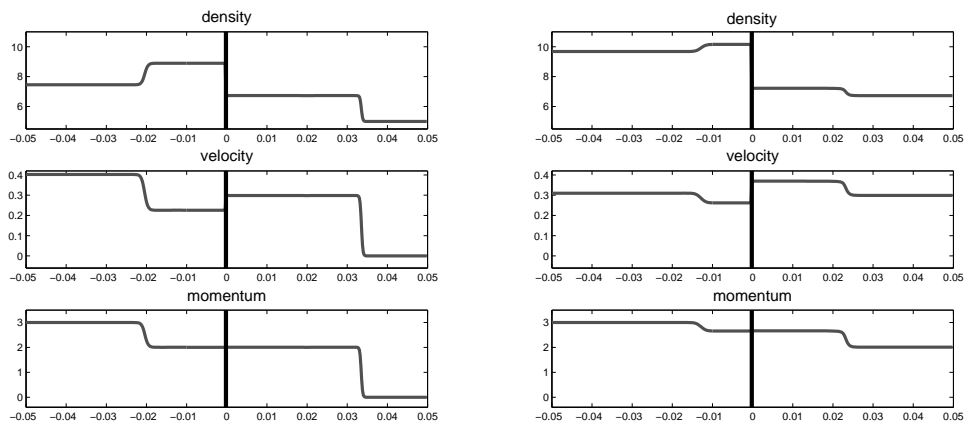

Fig. 3 Two successive interactions between shocks and particle in a clogged organ pipe

the pipe, with high density and low velocity before the particle, and low density and high velocity afterwards. Shapes of the solution after the first two interactions of a shock with the particle are depicted on Figure 3. This simulation illustrates the convergence of the ghost fluid scheme (4) on Riemann problem. We used the Godunov numerical flux but the results are similar with the Rusanov flux.

\subsection{Dealing with a moving particle}

We now focus on the case where the particle is free to move under the influence of the fluid. We saw in the introduction that it was necessary to treat the particle as an interface. Therefore, the particle must end up at an interface between two cells at the end of each time iteration. We could have used a mesh tracking the particle, but with in mind more complex applications (with numerous particles for example) we decided to use a fixed mesh and a Glimm's approach to replace the particle. At each time iteration, a real number $x_{r}$ is uniformly picked up in $[0, \Delta x]$. In the $j$-th cell, the fluid is updated by the exact value of the solution at time $\Delta t$ and at point $x_{r}$ of the Euler equation with initial data

$$
U^{0}(x)=U_{j-1}^{n} \mathbf{1}_{x<0}+U_{j}^{n} \mathbf{1}_{0<x<\Delta x}+U_{j+1}^{n} \mathbf{1}_{\Delta x<x} .
$$

Under the classical CFL condition $\Delta t<\frac{\Delta x}{2 \max _{x}|u(x)|+c}$, the solution consists in the juxtaposition of two Riemann problems. When $j$ corresponds to a neighbor cell of the particle, one of these Riemann problems takes the particle into account. The particle's position is updated in accordance to $x_{r}$. If the particle it at the interface $j_{0}^{n}+1 / 2$ at time $n$, and has speed $v^{n}$, then at time $n+1$ we placed it:

1. at interface $j_{0}^{n}+3 / 2$ if $v^{n}>0$ and $x_{r}<v^{n} \Delta t$, in which case $j_{0}^{n+1}=j_{0}^{n}+1$;

2. at interface $j_{0}^{n}-1 / 2$ if $v^{n}<0$ and $x_{r}>\Delta x+v^{n} \Delta t$, in which case $j_{0}^{n+1}=j_{0}^{n}-1$;

3. at interface $j_{0}^{n}+1 / 2$ otherwise, in which case $j_{0}^{n+1}=j_{0}^{n}$; 
Eventually, we update the particle's velocity using (2) and the numerical traces. The following numerical simulation is inspired by [6]. A marble falls into a cylinder filled with a compressible inviscid gas, which is initially at rest and of density $1 / 225 \mathrm{~kg} / \mathrm{m}$. Both the gas and the marble are subject to gravity and friction. The complete system writes

$$
\left\{\begin{array}{l}
\partial_{t} \rho+\partial_{x}(\rho u)=0 \\
\partial_{t}(\rho u)+\partial_{x}\left(\rho u^{2}+c^{2} \rho\right)=-\lambda\left(u-h^{\prime}\right) \delta_{h(t)}(x)-\rho g-v_{F}(\rho, u) \\
m h^{\prime \prime}(t)=\lambda\left(u(t, h(t))-h^{\prime}(t)\right)-m g-m v_{S}\left(h^{\prime}(t)\right)
\end{array}\right.
$$

where we take as in [6], $v_{S}\left(h^{\prime}\right)=10^{-2} h^{\prime}, v_{F}(\rho, u)=10^{-8} \rho u|u|, c=15 \mathrm{~m} / \mathrm{s}, \mathrm{m}=$ $0.004 \mathrm{~kg}$ and $\mathrm{g}=9.81 \mathrm{~m} / \mathrm{s}^{2}$. We took $\lambda=5 \mathrm{~m}^{2} \cdot \mathrm{kg} / \mathrm{s}$. The first term of the ODE
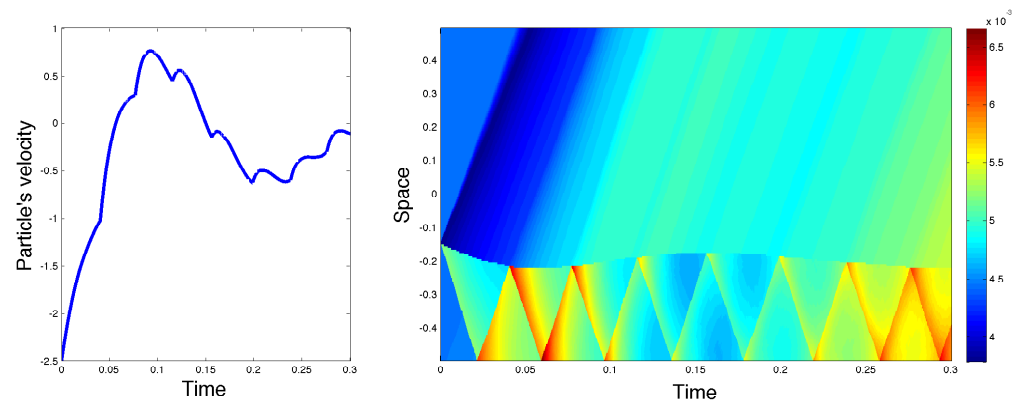

Fig. 4 Left: velocity of the particle. Each discontinuity on its acceleration is caused by a shock hitting the marble. Right: density of the fluid on the tube through time. We can see the shock with decreasing strength trapped between the marble and the bottom of the tube.

should be understood as in Equation (2). At first, the marble compresses the gas beneath it, creating a shock, and its velocity decreases due to friction. At some time, the shock reflects on the closed bottom of the tube, and then hits the marble, creating a discontinuity in its acceleration and accelerating it. This can be seen on Figure 4, on the left. When the shock interacts with the marble, it is somehow split in two: a part is reflected downward and a part passes through the marble and exits freely on the top end of the tube. Therefore the shock trapped between the marble and the bottom of the tube is of decreasing strength, as it can be seen on the plot of the density on the right of Figure 4 . The particle being very light, it is very sensible to the fluid's velocity, which is positive when the first shocks are moving upward. It causes the marble to climb back up for a while, then the gravity becomes predominant and the marble falls down again. The results are qualitatively the same as in [6]. However, they do not match perfectly, because the modeling is quite different. In particular in [6], the friction between the fluid and the marble is taken into account via a source term $v_{I}=5\left(h^{\prime}-\frac{u_{-}+u_{+}}{2}\right)^{2}$, while it is modeled directly through the interface conditions of Definition 1 in the present work. 


\section{Perspectives}

Let us start with some remarks on System (1), for which we proved in [2] existence and uniqueness to the Riemann problem when the particle is motionless, and give in this paper some qualitative properties and illustrative numerical simulations. Further theoretical study of System (1) seems difficult, as we have to deal with a system which is neither conservative nor hyperbolic. Even the extension of Theorem 1 to a freely moving particle is tricky, because the solution is not self-similar, and the traces around the particle constantly change. It is not difficult to extend the result to other pressure law, at least to $p(\rho)=a \rho^{\gamma}$, with $1<\gamma \leq 3, a>0$ and where no vacuum appears. Therefore, this model could be used to model the influence of an obstacle into the shallow water equation. Similarly, the extension to the full Euler equations is interesting, and could take into account exchange of heat between the fluid and the particle.

\section{References}

1. Abgrall, R., Karni, S.: Computations of compressible multifluids. J. Comput. Phys. 169(2) (2001)

2. Aguillon, N.: Riemann problem for a fluid-particule coupling. Submitted (2014)

3. Andreianov, B., Karlsen, K.H., Risebro, N.H.: A theory of $L^{1}$-dissipative solvers for scalar conservation laws with discontinuous flux. Arch. Ration. Mech. Anal. 201 (2011)

4. Andreianov, B., Lagoutière, F., Seguin, N., Takahashi, T.: Well-posedness for a onedimensional fluid-particle interaction model. To appear in SIAM J. Appl. Math. 5 (2013)

5. Andreianov, B., Seguin, N.: Analysis of a Burgers equation with singular resonant source term and convergence of well-balanced schemes. Discrete Contin. Dyn. Syst. 32 (2012)

6. Borsche, R., Colombo, R.M., Garavello, M.: On the interactions between a solid body and a compressible inviscid fluid. To appear in Interfaces Free Bound. (2014)

7. Boutin, B., Coquel, F., LeFloch, P.G.: Coupling techniques for nonlinear hyperbolic equations. III. The well-balanced approximation of thick interfaces. SIAM J. Numer. Anal. 51 (2013)

8. Colombo, R.M., Guerra, G.: On general balance laws with boundary. J. Differential Equations $248(2010)$

9. Dal Maso, G., Lefloch, P.G., Murat, F.: Definition and weak stability of nonconservative products. J. Math. Pures Appl. (9) 74(6) (1995)

10. Fedkiw, R.P., Aslam, T., Merriman, B., Osher, S.: A non-oscillatory Eulerian approach to interfaces in multimaterial flows (the ghost fluid method). J. Comput. Phys. 152 (1999)

11. Goatin, P., LeFloch, P.G.: The Riemann problem for a class of resonant hyperbolic systems of balance laws. Ann. Inst. H. Poincaré Anal. Non Linéaire 21 (2004)

12. Greenberg, J.M., Leroux, A.Y.: A well-balanced scheme for the numerical processing of source terms in hyperbolic equations. SIAM J. Numer. Anal. 33(1) (1996)

13. Hayes, B.T., Lefloch, P.G.: Nonclassical shocks and kinetic relations: strictly hyperbolic systems. SIAM J. Math. Anal. 31(5) (2000)

14. Isaacson, E., Temple, B.: Nonlinear resonance in systems of conservation laws. SIAM J. Appl. Math. 52 (1992)

15. Lagoutière, F., Seguin, N., Takahashi, T.: A simple 1D model of inviscid fluid-solid interaction. J. Differential Equations 245 (2008)

16. Parés, C.: Numerical methods for nonconservative hyperbolic systems: a theoretical framework. SIAM J. Numer. Anal. 44 (2006) 